\title{
Electron-Phonon Interaction in Embedded Semiconductor Nanostructures
}

\author{
Frank Grosse ${ }^{*}$ and Roland Zimmermann \\ Institut für Physik der Humboldt-Universität zu Berlin, Newtonstr. 15, 12489 Berlin, Germany
}

(Dated: October 1, 2018)

\begin{abstract}
The modification of acoustic phonons in semiconductor nanostructures embedded in a host crystal is investigated including corrections due to strain within continuum elasticity theory. Effective elastic constants are calculated employing $a b$ initio density functional theory. For a spherical InAs quantum dot embedded in GaAs barrier material, the electron-phonon coupling is calculated. Its strength is shown to be suppressed compared to the assumption of bulk phonons.

PACS numbers: $63.22 .+\mathrm{m}, 63.20 . \mathrm{Kr}, 63.20 . \mathrm{Ry}, 71.38 .-\mathrm{k}$
\end{abstract}

\section{INTRODUCTION}

Semiconductor nanostructures have attracted increasing interest over the last couple of years. Especially quantum dots (QD) are proposed as possible candidates in applications like single photon sources and quantum information devices. Improved growth techniques nowadays enable the fabrication of small QDs with a narrow size distribution. Single, isolated QDs embedded in a semiconductor matrix or in solution are of high interest for basic research. Since their electronic properties are inevitable connected to the underlying lattice and its vibrations (phonons) they offer the possibility to study these fundamental interactions. While the theory for electron-phonon interaction in bulk systems is well established, the inhomogeneous nature of nanostructures leads to strong modifications of the electronic properties as well as the phonon spectrum. Usually, QDs are designed such that at least the energetically lowest state is spatially confined. The existence of boundaries between the constituting materials and/or to the vacuum introduces a coupling of the longitudinal and transverse phonon modes even for isotropic media. Additionally, new types of confined interface and surfaces modes can occur. Especially the electron-acoustic phonon interaction is of high interest since it is the source of so-called pure dephasing of optical excitations in QDs, as detailed in recent articles 1.2 .

The electron-acoustic phonon interaction is usually treated by deformation potential coupling, and acoustic phonons are described as bulk phonons. Only recently some progress has been made to include phonons in inhomogeneous media in the treatment of electron-phonon interaction in slab and half space geometries ${ }^{1}$. So far to the best of our knowledge no investigations exist treating the modified phonons of the nanostructure itself in the interaction.

The present article provides insights into the role of the phonon modification due to the nanostructure, including strain. We restrict ourselves to the simplest case - a spherical QD - in order to describe the qualitative effect on the electron-acoustic phonon coupling. The details of the model and the corresponding theory are developed in Sec. III Calculations of strain dependent acoustic phonons on the basis of $a b$ initio density functional theory (DFT) calculations are given in Sec. IIB which serve as input for the calculation of the electron-phonon coupling function. Results are given in Sec. III]

\section{MODEL AND THEORY}

Epitaxial growth methods enable the fabrication of semiconductor heterostructures with sizes of a few nanometers only. The different materials are connected pseudomorphically, i.e. without structural defects. Defects would lead to partial relaxation of intrinsic strain which results from different lattice constants of the constituents. The strain itself causes the change of physical properties compared to relaxed bulk materials. This includes the electronic (band structure) as well as the lattice properties (phonons).

The system we study in this article is a spherical shape inclusion (quantum dot) in an infinite matrix (barrier). This artificial system should serve as test model for the relevance of non-bulk phonon modes in the electronphonon coupling. For the material parameters we choose the prototype InAs/GaAs system. The first part is devoted to the description of the acoustic phonons within continuum elasticity theory followed by the description of the electron-acoustic phonon coupling.

\section{A. Acoustic phonons within continuum elasticity}

In elastic continuum theory, acoustic phonons follow from the wave equation of the displacement field $\mathbf{u}(\mathbf{r})$

$$
-\omega^{2} \rho(\mathbf{r}) u_{j}(\mathbf{r})=\sum_{k} \frac{\partial}{\partial x_{k}} \sigma_{j k}(\mathbf{r}),
$$

here written in Cartesian coordinates $j, k=x, y, z$. Both the mass density $\rho(\mathbf{r})$ and the stress tensor $\stackrel{\leftrightarrow}{\sigma}(\mathbf{r})$ are in general spatially dependent. In linear elasticity theory the stress tensor $\overleftrightarrow{\sigma}$ is proportional to the strain tensor $\stackrel{\leftrightarrow}{\epsilon}$ defined by the spatial derivatives of the displacement fields, both quantities being related by the fourth order tensor of elastic constants $\stackrel{\leftrightarrow}{C}$ (Hooke's law). For isotropic 
systems there are only two independent elastic constants $C_{11}$ and $C_{44}$, which are related to the well-known Lamé coefficients $C_{12}=C_{11}-2 C_{44}=\lambda$ and $C_{44}=\mu$. The stress tensor elements are then given by

$\sigma_{j k}=\delta_{j k}\left(C_{11}-2 C_{44}\right) \sum_{l} \frac{\partial u_{l}}{\partial x_{l}}+C_{44}\left(\frac{\partial u_{j}}{\partial x_{k}}+\frac{\partial u_{k}}{\partial x_{j}}\right)$

The restriction to isotropic media allows to treat the phonons in large part analytically.

For our spherical model system it is convenient to transform to spherical coordinates. For the specific electron-phonon coupling considered below, the only relevant phonon mode has angular momentum zero (breathing mode), $\mathbf{u}(\mathbf{r})=\mathbf{e}_{r} u(r)$, which fulfills the equation

$$
\begin{aligned}
0= & \rho(r) \omega^{2} u(r)-4 \frac{d C_{44}(r)}{d r} \frac{u(r)}{r} \\
& +\frac{d}{d r}\left[C_{11}(r)\left(\frac{d u(r)}{d r}+\frac{2 u(r)}{r}\right)\right]
\end{aligned}
$$

with elastic constants and mass density having an arbitrary radial dependence. This one-dimensional secondorder differential equation contains the boundary conditions for a stepwise constant medium as well, which are

$$
u \text { and } C_{11}\left(\frac{d u}{d r}+\frac{2 u}{r}\right)-4 C_{44} \frac{u}{r} \text { continuous }
$$

We solve Eq. (3) numerically, starting with a regular solution $u(r) \propto r$ at the origin. The general normalization condition of the displacement within a large volume $\Omega$ reads 3

$$
\int_{\Omega} d^{3} r \rho(\mathbf{r}) \mathbf{u}_{\nu}(\mathbf{r}) \cdot \mathbf{u}_{\nu^{\prime}}(\mathbf{r})=\delta_{\nu, \nu^{\prime}} \frac{\hbar}{2 \omega_{\nu}}
$$

where $\nu$ is the discrete mode index. This normalization is converted into a matching condition at large distances with an outgoing wave containing a phase shift, as standard in scattering theory. As a consequence, $\nu$ is replaced by a (continuous) energy variable $\hbar \omega_{\nu} \rightarrow E$.

The elastic constants in Eq. (3) are not to be taken as the usual tabulated bulk values for the dot and the barrier material: The phonon calculation has to start from the reference state where the crystal is locally under finite strain. Therefore the spatial dependency is not only due to the change in material but also due to the spatially varying strain.

\section{B. Effective Elastic Constants from DFT}

The usual treatment of continuum elasticity assumes fixed elastic constants. In our case, however, the system contains spatial regions where the material is strained. Intuitively, one would expect that a material under compression would become harder due to the presence of an-

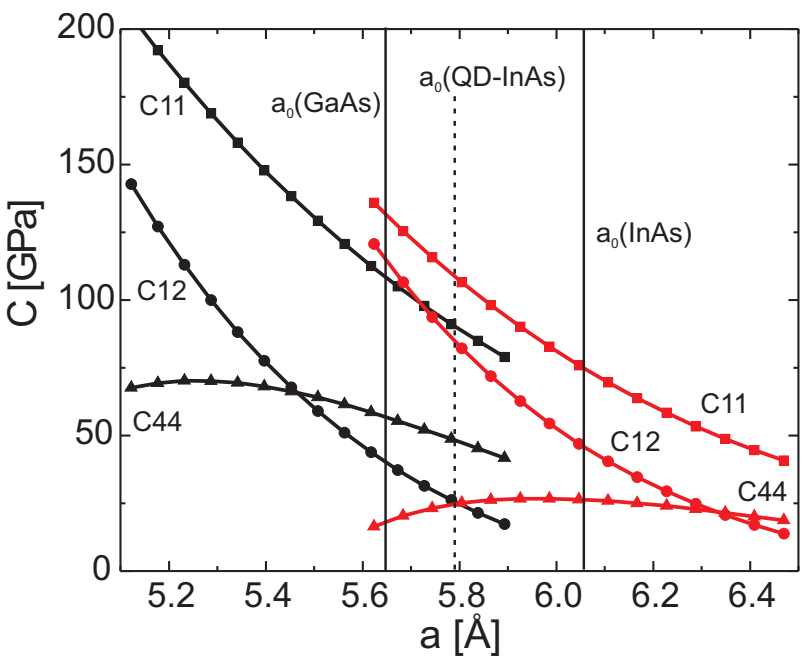

FIG. 1: Effective elastic constants for GaAs (black) and InAs (red) in dependence on the lattice constant $a$ for hydrostatic deformations calculated by DFT employing perturbation theory. The (experimental) equilibrium lattice constants are marked by full vertical lines, while the lattice constant of the relaxed InAs QD is given by the dashed line.

harmonic terms in the expansion of the free energy density around the equilibrium position,

$$
\rho_{0} \phi=\frac{1}{2} \sum_{I, J=1}^{6} C_{I J} \epsilon_{I} \epsilon_{J}+\frac{1}{3 !} \sum_{I, J, K=1}^{6} C_{I J K} \epsilon_{I} \epsilon_{J} \epsilon_{K}+\ldots
$$

The equilibrium density is written as $\rho_{0}$. We restrict ourselves to hydrostatic compressions in the following. An extension to general deformations is straightforward but numerically a far more complex task. Allowing only small distorsions of the lattice (an expansion up to second order is sufficient), only the bulk modulus $B=1 / 3\left(C_{11}+\right.$ $2 C_{12}$ ) would be a constant quantity. Including up to third order in the expansion an analytical derivation gives a linear dependence on the strength of the hydrostatic lattice distortion ${ }^{4}$.

The calculation of elastic constants is possible directly by employing DFT. Starting from a relaxed unit cell under the application of finite deformations within density functional perturbation theory one can calculate the fourth order tensor of elastic constants. The density functional calculations are carried out with the ABINIT computer code ${ }^{5.6 .7}$. The local density approximation is applied for the exchange-correlation. Soft norm-conserving pseudopotentials are taken from the code of the FritzHaber Institute, Berlin ${ }^{8}$. Wave functions are expanded into plane waves with converged Monkhorst-Pack meshes of $k=8 \times 8 \times 8$ per $1 \times 1$ unit cell and a cutoff energy of $E_{c u t}=36 \mathrm{Ha} \equiv 979 \mathrm{eV}$.

The dependence of the calculated elastic constants on the (variable) lattice constant, which corresponds to hydrostatic deformation, is displayed in Fig. 11 In the range from $-7 \%$ to $+7 \%$ lattice constant variation we find a 
nearly linear reduction of $C_{11}$ and $C_{12}$ with increasing the lattice constant, which is expected from a third order expansion ${ }^{4}$, showing that even for small distorsions the assumption of constant elasticity does not hold. The deviation from the linear behavior, especially for $C_{44}$, is a clear indication that even higher orders than the third one influence significantly the effective elastic constants. Numerical deviations from the experimental values are mainly due to the known underestimation of the lattice constant in LDA for III-V semiconductors (see Tab. 【). Given a local static lattice distortion, it is now possible to implement these elastic constants in the phonon calculation, which depend on the spatial position due to change in material and strain as well.

\section{Electron-acoustic phonon coupling}

The electron-phonon interaction has two main contributions: Deformation potential coupling and piezoelectric coupling. The second contribution will not be considered here, although some of the following discussions apply also to this part.

For conduction electrons at the $\Gamma$-point in III-V semiconductors the deformation potential couples only to a volume deformation ( $\operatorname{div} \mathbf{u})$. The interaction Hamiltonian is ${ }^{3}$

$$
H_{\mathrm{def}}(\mathbf{r})=\sum_{\nu} D_{C}(\mathbf{r})\left[b_{\nu}^{\dagger} \operatorname{div} \mathbf{u}_{\nu}(\mathbf{r})+\text { h.c. }\right]
$$

with the (material-dependent) deformation potential constant for the conduction band $D_{C}$ and the phonon creation operator $b_{\nu}^{\dagger}$. In a QD with confinement functions $\varphi_{n}(\mathbf{r})$ of the electron, the relevant electron-phonon matrix elements are then

$$
M_{\nu}^{n m}=\int d^{3} r \varphi_{n}^{*}(\mathbf{r}) \varphi_{m}(\mathbf{r}) D_{C}(\mathbf{r}) \operatorname{div} \mathbf{u}_{\nu}(\mathbf{r}) .
$$

The spherical symmetry of the QD leads to well-defined angular quantum numbers of the confinement levels, which select an appropriate symmetry of the lattice displacement. For electronic s-s-transitions, only the azimuthal quantum number $l=0$ is needed (breathing mode). For the polaronic modification of a single electronic transition within the independent Boson mode ${ }^{\underline{9}}$, the diagonal element in Eq. (17) is of central importance. Concentrating on the lowest (s-type) confinement state $n=1$, we need to calculate the following coupling func$\operatorname{tion}^{10}$

$$
f(E)=\sum_{\nu} \delta\left(E-\hbar \omega_{\nu}\right)\left|M_{\nu}^{11}\right|^{2} .
$$

For instance, the so-called broad band around the zerophonon line at $E=0$ has a shape close to $f(E) / E^{3}$ at elevated temperatures (strictly speaking, the hole confinement state participating in the transition has to be included as well).

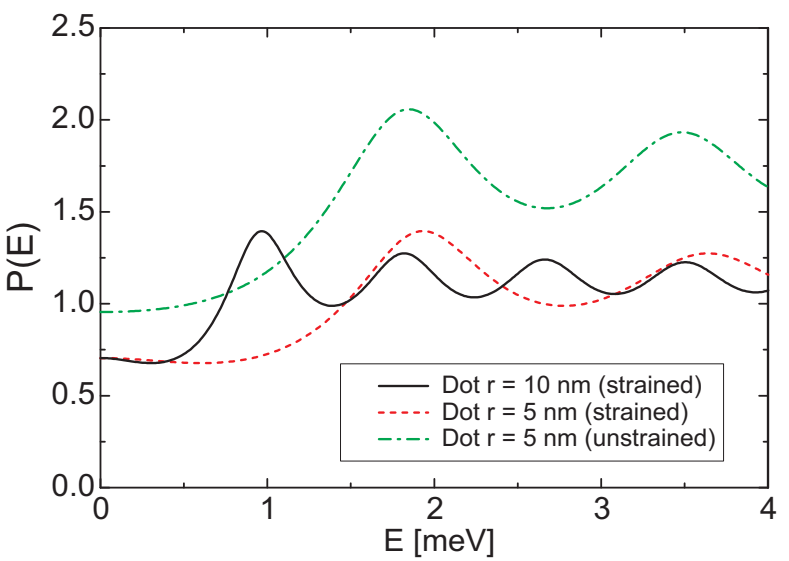

FIG. 2: Energy dependence of the phonon amplitudes for an InAs sphere of radius $R$ embedded into GaAs material. The unrealistic situation without strain is compared with the strained one.

\section{RESULTS AND DISCUSSION}

In the following we present results for a spherical QD consisting of pure InAs embedded in infinitely extended GaAs. Under hydrostatic compression the coordinates change according to $x_{j}^{\prime}=(1-\alpha) x_{j}$. The deformation $\alpha$ can be determined from the static solution $(\omega=0)$ of Eq. 3 Applying the boundary conditions from Eq. 4 accordingly leads to

$$
\alpha=\alpha_{0} \frac{C_{44, \mathrm{GaAs}}}{C_{44, \mathrm{InAs}}-C_{44, \mathrm{GaAs}}+\frac{3}{4} C_{11, \mathrm{InAs}}} .
$$

with the lattice mismatch $\alpha_{0}$. For the present InAs/GaAs model system, it is $\alpha_{0}=\left(a_{0, \text { InAs }}-a_{0, \mathrm{GaAs}}\right) / a_{0, \mathrm{InAs}}=$ $6.8 \%$ using the experimental values given in Tab. The relaxed lattice constant $a$ of the dot material (InAs), which is compressed hydrostatically, is therefore $a_{\mathrm{InAs}}=$ $a_{0, \text { InAs }}(1-\alpha)$ (last row of Tab. \ and indicated in Fig. \ as dashed line.)

Outside the dot, the material is compressed in radial direction but dilated laterally resulting in a zero net volume change. Since we consider only hydrostatic compression the elastic properties are taken unchanged for the barrier material.

We start by looking at the common approximation of using bulk phonons of the barrier material (index B) throughout. The solution of Eq. (33) gives simply

$$
u^{(B)}(r)=j_{1}\left(k_{B} r\right)
$$

with $j_{l}(x)$ being the spherical Bessel function of the first kind, and the wave number is related to the energy via $E=\hbar v_{l B} k_{B}\left(v_{l}=\sqrt{C_{11} / \rho}\right.$ is the longitudinal sound velocity). Insertion into Eq. (8) leads to

$$
f^{(B)}(E)=\frac{E^{3} D_{C B}^{2}}{4 \pi^{2} \hbar^{3} \rho_{B} v_{l B}^{5}}\left|\int_{0}^{\infty} d r r^{2} \varphi_{1}^{2}(r) j_{0}\left(k_{B} r\right)\right|^{2} .
$$



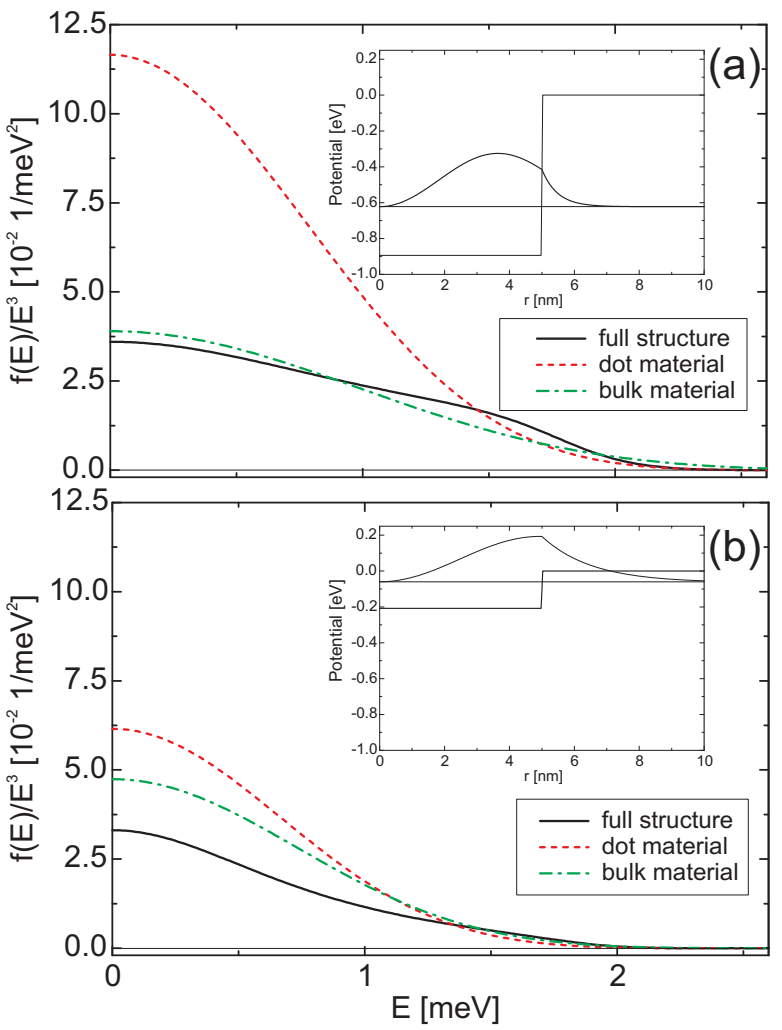

FIG. 3: Electron-acoustic phonon coupling function. Compared are (a) the unstrained combination of an InAs QD $(R=5 \mathrm{~nm})$ embedded in GaAs and the more realistic (b) strained situation. In each panel the assumption of bulk phonons, either GaAs or InAs, is compared to the full phonon calculation. The inset shows the electron band offset and the confinement charge density.

In this approximation, the coupling function (divided by $E^{3}$ ) follows the (squared) Fourier transform of the electron charge density, which decays on a scale of $E \approx$ $\hbar v_{l B} / R$ ( $R$ is the $\mathrm{QD}$ radius).

For the full problem, the displacement inside the dot (index D) is getting a prefactor,

$$
u(r)=A(E) j_{1}\left(k_{D} r\right),
$$

which has to be determined from solving Eq. 3 numerically. Although the integration in the matrix element extends into the barrier material as well, it is mainly the variation of $A(E)$ which modifies the coupling function. In order to implement the change in wave number (sound velocity) as well, we found it convenient to define a phonon amplitude as

$$
P(E)=A(E) \frac{v_{l B}}{v_{l D}} .
$$

By definition, for bulk barrier phonons, $P(E) \equiv 1$ which is the normalization used in Fig. 2

Whereas for a free standing sphere as well as for a rigidly clamped sphere, only discrete phonon energies are allowed, the QD embedded in an infinite elastic medium

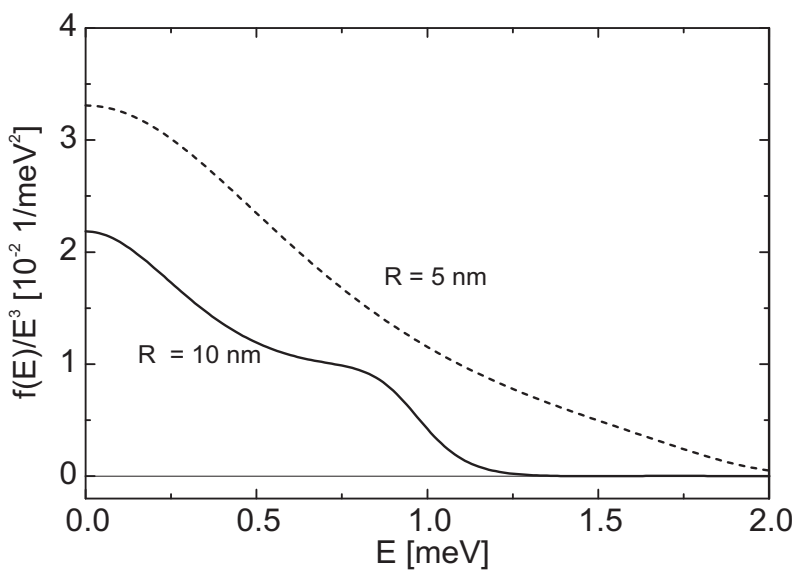

FIG. 4: Electron-acoustic phonon coupling function: Compared are two different radii of the strained InAs QD embedded in GaAs.

\begin{tabular}{c|cccccc} 
& $\begin{array}{c}m_{e} \\
{\left[m_{0}\right]}\end{array}$ & $\begin{array}{c}D_{C} \\
{[\mathrm{eV}]}\end{array}$ & $\begin{array}{c}\mathrm{CBO} \\
{[\mathrm{meV}]}\end{array}$ & $\begin{array}{c}\rho \\
{\left[\mathrm{g} / \mathrm{cm}^{3}\right]}\end{array}$ & $a_{0}(\mathrm{Exp})$ & $a_{0}(\mathrm{DFT})$ \\
{$[\AA]$} & {$[\AA]$} \\
\hline GaAs & 0.067 & -7.17 & 0 & 5.33 & 5.65 & 5.51 \\
$\mathrm{InAs}$ & 0.026 & -5.08 & -894 & 5.66 & 6.06 & 6.05 \\
\hline s-InAs & 0.026 & -5.08 & -208 & 6.50 & 5.79 & -
\end{tabular}

TABLE I: Parameters used in the calculation for the unstrained material11. Given are also the theoretical lattice constants $a_{0}$ (DFT). Last row: Resulting values for the embedded strained InAs quantum dot (s-InAs).

allows for a continuous manifold of phonon energies. However, the oscillating behavior of the phonon amplitude shown in Fig. 2 resembles the eigenmodes of an isolated sphere. A specific feature is the reduction of the phonon amplitude at small energies: Including the strain, its overall strength is reduced even further. Doubling the size of the embedded dot from $R=5 \mathrm{~nm}$ to $R=10 \mathrm{~nm}$ leads to halve the energetic spacing between maxima, while the overall amplitude remains the same. This can be understood by noting that within the applied approximation, neither the static strain field nor the material density depends on the size of the QD.

The calculation of the electron confinement wave function is done within the effective mass approximation including mass discontinuities. All relevant input variables are taken from existing literature ${ }^{11}$ and given in Tab.[ The compressive strain in the InAs dot reduces the conduction band offset (CBO) significantly. The resulting electron confinement potential is plotted in the insets of Fig. [3 together with the charge density in the lowest confinement state, $r^{2} \varphi_{1}^{2}(r)$. In the strained case (Fig. 3b), a larger tunneling of the wave function into the barrier can be seen. However, this change in the electronic wave function affects the electron-phonon coupling only marginal.

The coupling function Eq. (8) of electrons with acoustic phonons is shown in Fig. 3 comparing the strain free case 
(a) with the improved description including the strain present in the nanostructure (b). Within each panel the approximation of bulk phonons using either barrier (GaAs) or dot (InAs) material parameter are displayed, too. Compared to 'dot-bulk phonons', a strong reduction is found, whereas 'barrier-bulk phonons' are much closer to the full solution, although missing the existing structure in the coupling function.

The compressive strain results in an increased mass density (see Tab. I), increased elastic constants in the dot and to a reduction of the coupling strength $\operatorname{div} \mathbf{u}(\mathbf{r})$. The shape of the coupling function, however, is not much changed when including strain since the maximum positions in the phonon amplitude are only slightly shifted. The coupling is reduced further by increasing the size of the QD, see Fig. [4. Here, the coupling function is even more structured (sharp drop at around $1 \mathrm{meV}$ ) which has its origin in the oscillatory variation of the phonon amplitude discussed before.

\section{CONCLUSIONS}

Including the details of the phonon characteristics in semiconductor nanostructures is essential for a quantita- tive description of the electron-acoustic phonon coupling. Material parameters, i.e. mass density and elastic constants, are changed due to intrinsic strain in pseudomorphically grown heterostructures and are therefore spatially dependent. Within continuum elasticity this can be treated by introducing effective material parameters. There is a continuous energy spectrum for phonons of nanostructures embedded in an infinite elastic medium. Compared to the assumption of bulk phonons, an overall reduction of the electron-acoustic phonon coupling is found due to the nanostructure as well as due to strain. Resonance-like features in the phonon modes lead to changes in the line shape of the energy dependent coupling function which are more pronounced for mid-size than for small dots.

\section{Acknowledgments}

The work has been done within the framework of SFB 296 of the Deutsche Forschungsgemeinschaft. Fruitful discussions with L. Wendler are acknowledged.
* Electronic address: frank.grosse@physik.hu-berlin.de URL: http://www-semic.physik.hu-berlin.de

1 B. Krummheuer, V. M. Axt, and T. Kuhn, Phys. Rev. B 72, 245336 (2005).

2 E. Muljarov and R. Zimmermann, Phys. Rev. Lett. 93, 237401 (2004).

3 C. Trallero-Giner, F. Comas, and F. García-Moliner, Phys. Rev. B 50, 1755 (1994).

4 S. Bosher and D. Dunstan, J. Appl. Phys. 97, 103505 (2005).

5 X. Gonze, J.-M. Beuken, R. Caracas, F. Detraux, M. Fuchs, G.-M. Rignanese, L. Sindic, M. Verstraete, G. Zerah, F. Jollet, et al., Computational Materials Science 25, 478 (2002).

${ }^{6}$ X. Gonze, G.-M. Rignanese, M. Verstraete, J.-M. Beuken,
Y. Pouillon, R. Caracas, F. Jollet, M. Torrent, G. Zerah, M. Mikami, et al., Zeit. Kristallogr. 220, 558 (2005).

7 The ABINIT code is a common project of the Université Catholique de Louvain, Corning Incorporated, and other contributors http://www.abinit.org. (v 4.6.5).

8 M. Fuchs and M. Scheffler, Comput. Phys. Commun. 116, 1 (1999).

${ }^{9}$ G. Mahan, Many-Particle Physics (Plenum, New York, 1990).

10 G. Mannarini and R. Zimmermann, Phys. Rev. B 73, 115325 (2006).

11 I. Vurgaftman, J. R. Meyer, and L. R. Ram-Mohan, J. Appl. Phys. 89, 5815 (2001). 